\title{
Partial sum of the products of the Horadam numbers with subscripts in arithmetic progression
}

\author{
Kunle Adegoke $^{1}$, Robert Frontczak ${ }^{2 *}$ and Taras Goy ${ }^{3}$ \\ ${ }^{1}$ Department of Physics and Engineering Physics, Obafemi Awolowo University \\ 220005 Ile-Ife, Nigeria \\ e-mail: adegoke00@gmail.com \\ ${ }^{2}$ Landesbank Baden-Württemberg \\ 70173 Stuttgart, Germany \\ e-mail: robert. frontczak@lbbw. de \\ ${ }^{3}$ Faculty of Mathematics and Computer Science \\ Vasyl Stefanyk Precarpathian National University \\ 76018 Ivano-Frankivsk, Ukraine \\ e-mail: taras.goy@pnu.edu.ua
}

Received: 8 February 2021

Revised: 3 May 2021

Accepted: 23 May 2021

Abstract: We evaluate the partial sum of the products of the terms of any two Horadam sequences with subscripts in arithmetic progression. Illustrative examples are drawn from six well-known Horadam sequences.

Keywords: Horadam sequence, Generating function, Fibonacci numbers, Lucas numbers, Pell numbers, Jacobsthal numbers, Pell-Lucas numbers, Jacobsthal-Lucas numbers.

2020 Mathematics Subject Classification: 11B37, 11B39.

\section{Introduction}

Our purpose in this paper is to evaluate the partial sum $\sum_{j=0}^{k} X_{r j+s}^{(1)} X_{m j+t}^{(2)} z^{j}$, where $\left(X_{n}^{(1)}\right)_{n \in \mathbb{Z}}$ and $\left(X_{n}^{(2)}\right)_{n \in \mathbb{Z}}$ are any two Horadam sequences, $r, s, m, t$ and $k$ are any integers and $z$ is any complex variable. Our results are related to those from [2-5,7-10].

${ }^{*}$ Statements and conclusions made in this paper by Robert Frontczak are entirely those of the author. They do not necessarily reflect the views of Landesbank Baden-Württemberg. 
The Horadam sequence [6] $\left(w_{n}\right)=\left(w_{n}(a, b ; p, q)\right)$ is defined, for all integers, by the recurrence relation

$$
w_{0}=a, w_{1}=b, \quad w_{n}=p w_{n-1}-q w_{n-2}, \quad n \geq 2,
$$

with

or, equivalently,

$$
w_{-n}=\frac{(a p-b) u_{n}-a q u_{n-1}}{q^{n}\left(b u_{n}-a q u_{n-1}\right)} w_{n}
$$

$$
w_{-n}=q^{-n}\left(a v_{n}-w_{n}\right),
$$

where $a, b, p$ and $q$ are arbitrary complex numbers, with $p \neq 0$ and $q \neq 0$; and $\left(u_{n}(p, q)\right)=$ $\left(w_{n}(0,1 ; p, q)\right)$ and $\left(v_{n}(p, q)\right)=\left(w_{n}(2, p ; p, q)\right)$ are Lucas sequences of the first kind and of the second kind, respectively. The most well-known Lucas sequences are the Fibonacci sequence $\left(F_{n}\right)=\left(u_{n}(1,-1)\right)$ and the sequence of Lucas numbers $\left(L_{n}\right)=\left(v_{n}(1,-1)\right)$.

The Binet formulas for $u_{n}, v_{n}$ and $w_{n}$ in the non-degenerate case $p^{2} \neq 4 q$ are

$$
u_{n}=\frac{\alpha^{n}-\beta^{n}}{\alpha-\beta}, \quad v_{n}=\alpha^{n}+\beta^{n}, \quad w_{n}=\frac{b-a \beta}{\alpha-\beta} \alpha^{n}+\frac{a \alpha-b}{\alpha-\beta} \beta^{n},
$$

where $\alpha$ and $\beta$ are the distinct zeros of the characteristic polynomial $x^{2}-p x+q$ of the Horadam and Lucas sequences,

$$
\alpha=\frac{p+\sqrt{p^{2}-4 q}}{2}, \quad \beta=\frac{p-\sqrt{p^{2}-4 q}}{2} .
$$

The following power reduction formulas, which we require later, can be easily established by induction:

$$
\alpha^{n}=u_{n} \alpha-q u_{n-1}, \quad \beta^{n}=u_{n} \beta-q u_{n-1} .
$$

Partial sum of Horadam numbers with subscripts in arithmetic progression for integers $r, k$ and $s$ and arbitrary $z$ can be evaluated as $[1,10]$

$$
\sum_{j=0}^{k} w_{r j+s} z^{j}=\frac{q^{r} w_{r k+s} z^{k+2}-w_{r k+r+s} z^{k+1}-q^{r} w_{s-r} z+w_{s}}{q^{r} z^{2}-v_{r} z+1} .
$$

In particular,

$$
\begin{aligned}
\sum_{j=0}^{k} u_{r j+s} z^{j} & =\frac{q^{r} u_{r k+s} z^{k+2}-u_{r k+r+s} z^{k+1}+q^{s} u_{r-s} z+u_{s}}{q^{r} z^{2}-v_{r} z+1}, \\
\sum_{j=0}^{k} v_{r j+s} z^{j} & =\frac{q^{r} v_{r k+s} z^{k+2}-v_{r k+r+s} z^{k+1}-q^{s} v_{r-s} z+v_{s}}{q^{r} z^{2}-v_{r} z+1} .
\end{aligned}
$$

The generating function of the Horadam sequence with subscripts in arithmetic progression for integers $r$ and $s$ is [1]

$$
\sum_{j=0}^{\infty} w_{r j+s} z^{j}=\frac{-q^{r} w_{s-r} z+w_{s}}{q^{r} z^{2}-v_{r} z+1}
$$

In particular,

$$
\sum_{j=0}^{\infty} u_{r j+s} z^{j}=\frac{q^{s} u_{r-s} z+u_{s}}{q^{r} z^{2}-v_{r} z+1}, \quad \sum_{j=0}^{\infty} v_{r j+s} z^{j}=\frac{-q^{s} v_{r-s} z+v_{s}}{q^{r} z^{2}-v_{r} z+1} .
$$


Further results on Horadam sequence can be found in the survey paper [13]. Properties of Lucas sequences can be found in [14, Chapter 1].

\section{Main results}

Theorem 2.1. Let $\left(X_{n}^{(1)}\right)=\left(w_{n}\left(X_{0}^{(1)}, X_{1}^{(1)} ; p_{1}, q_{1}\right)\right)$ and $\left(X_{n}^{(2)}\right)=\left(w_{n}\left(X_{0}^{(2)}, X_{1}^{(2)} ; p_{2}, q_{2}\right)\right)$ be two non-degenerated Horadam sequences. Let

$$
\begin{aligned}
& \left\{\left(u_{n}^{(1)}\right)=\left(w_{n}\left(0,1 ; p_{1}, q_{1}\right)\right),\left(v_{n}^{(1)}\right)=\left(w_{n}\left(2, p_{1} ; p_{1}, q_{1}\right)\right)\right\}, \\
& \left\{\left(u_{n}^{(2)}\right)=\left(w_{n}\left(0,1 ; p_{2}, q_{2}\right)\right),\left(v_{n}^{(2)}\right)=\left(w_{n}\left(2, p_{2} ; p_{2}, q_{2}\right)\right)\right\}
\end{aligned}
$$

be the respective Lucas sequences associated with $\left(X_{n}^{(1)}\right)$ and $\left(X_{n}^{(2)}\right)$. Then

$$
\sum_{j=0}^{k} X_{r j+s}^{(1)} X_{m j+t}^{(2)} z^{j}=\frac{X_{0}^{(2)}\left(q_{2} E G+p_{2} F G+F H\right)+X_{1}^{(2)}(E H-F G)}{q_{2} G^{2}+H^{2}+p_{2} G H},
$$

where

$$
\begin{gathered}
E=q_{1}^{r} X_{r k+s}^{(1)} u_{m k+2 m+t}^{(2)} z^{k+2}-X_{r k+r+s}^{(1)} u_{m k+m+t}^{(2)} z^{k+1}-q_{1}^{r} X_{s-r}^{(1)} u_{m+t}^{(2)} z+X_{s}^{(1)} u_{t}^{(2)}, \\
F=-q_{1}^{r} X_{r k+s}^{(1)} u_{m k+2 m+t-1}^{(2)} z^{k+2}+X_{r k+r+s}^{(1)} u_{m k+m+t-1}^{(2)} z^{k+1} \\
+q_{1}^{r} X_{s-r}^{(1)} u_{m+t-1}^{(2)} z-X_{s}^{(1)} u_{t-1}^{(2)} \\
G=q_{1}^{r} u_{2 m}^{(2)} z^{2}-v_{r}^{(1)} u_{m}^{(2)} z
\end{gathered}
$$

and

$$
H=-q_{1}^{r} q_{2} u_{2 m-1}^{(2)} z^{2}+q_{2} v_{r}^{(1)} u_{m-1}^{(2)} z+1 .
$$

Proof. Let $\alpha_{i}$ and $\beta_{i}, \alpha_{i} \neq \beta_{i}, i \in\{1 ; 2\}$, be the zeros of $x^{2}-p_{i} x+q_{i}$, the characteristic polynomial of the sequence $\left(u_{n}^{(i)}\right)$. Then

$$
X_{n}^{(i)}=A_{i} \alpha_{i}^{n}+B_{i} \beta_{i}^{n}, \quad i \in\{1 ; 2\},
$$

where

$$
A_{i}=\frac{X_{1}^{(i)}-X_{0}^{(i)} \beta_{i}}{\alpha_{i}-\beta_{i}}, \quad B_{i}=\frac{X_{0}^{(i)} \alpha_{i}-X_{1}^{(i)}}{\alpha_{i}-\beta_{i}} .
$$

In (2) make the identification $w_{n} \equiv X_{n}^{(1)}$, replace $z$ with $\alpha_{2}^{m} z$ and multiply both sides by $\alpha_{2}^{t}$ to obtain

$$
\begin{aligned}
S_{1} & =\sum_{j=0}^{k} X_{r j+s}^{(1)} \alpha_{2}^{m j+t} z^{j} \\
& =\frac{q_{1}^{r} X_{r k+s}^{(1)} \alpha_{2}^{m k+2 m+t} z^{k+2}-X_{r k+r+s}^{(1)} \alpha_{2}^{m k+m+t} z^{k+1}-q_{1}^{r} X_{s-r}^{(1)} \alpha_{2}^{m+t} z+X_{s}^{(1)} \alpha_{2}^{t}}{q_{1}^{r} \alpha_{2}^{2 m} z^{2}-v_{r}^{(1)} \alpha_{2}^{m} z+1} .
\end{aligned}
$$

Similarly,

$$
\begin{aligned}
S_{2} & =\sum_{j=0}^{k} X_{r j+s}^{(1)} \beta_{2}^{m j+t} z^{j} \\
& =\frac{q_{1}^{r} X_{r k+s}^{(1)} \beta_{2}^{m k+2 m+t} z^{k+2}-X_{r k+r+s}^{(1)} \beta_{2}^{m k+m+t} z^{k+1}-q_{1}^{r} X_{s-r}^{(1)} \beta_{2}^{m+t} z+X_{s}^{(1)} \beta_{2}^{t}}{q_{1}^{r} \beta_{2}^{2 m} z^{2}-v_{r}^{(1)} \beta_{2}^{m} z+1} .
\end{aligned}
$$


Using the power reduction formulas (1), identities (7) and (8) can be written as

$$
S_{1}=\sum_{j=0}^{k} X_{r j+s}^{(1)} \alpha_{2}^{m j+t} z^{j}=\frac{E \alpha_{2}+F}{G \alpha_{2}+H}
$$

and

$$
S_{2}=\sum_{j=0}^{k} X_{r j+s}^{(1)} \beta_{2}^{m j+t} z^{j}=\frac{E \beta_{2}+F}{G \beta_{2}+H},
$$

where $E, F, G$ and $H$ are as given in (3)-(6). Now

$$
A_{2} S_{1}+B_{2} S_{2}=\sum_{j=0}^{k} X_{r j+s}^{(1)}\left(A_{2} \alpha_{2}^{m j+t}+B_{2} \beta_{2}^{m j+t}\right) z^{j}=\sum_{j=0}^{k} X_{r j+s}^{(1)} X_{m j+t}^{(2)} z^{j} .
$$

But from (9) and (10) we have

$$
A_{2} S_{1}+B_{2} S_{2}=A_{2} \frac{E \alpha_{2}+F}{G \alpha_{2}+H}+B_{2} \frac{E \beta_{2}+F}{G \beta_{2}+H} .
$$

Thus,

$$
\sum_{j=0}^{k} X_{r j+s}^{(1)} X_{m j+t}^{(2)} z^{j}=\frac{A_{2}\left(E \alpha_{2}+F\right)\left(G \beta_{2}+H\right)+B_{2}\left(G \alpha_{2}+H\right)\left(E \beta_{2}+F\right)}{\left(G \alpha_{2}+H\right)\left(G \beta_{2}+H\right)},
$$

from which the stated identity follows after multiplying out the right-hand side and some algebra.

\section{Examples}

We will draw illustrations of Theorem 2.1 from six well-known second-order sequences, namely the Fibonacci, Lucas, Pell, Pell-Lucas, Jacobsthal, and Jacobsthal-Lucas numbers. First we give a quick review of the sequences.

The Fibonacci numbers $F_{n}$ and the Lucas numbers $L_{n}$ are defined, for $n \in \mathbb{Z}$, as usual, through the recurrence $F_{n}=F_{n-1}+F_{n-2}(n \geq 2), F_{0}=0, F_{1}=1$ and $L_{n}=L_{n-1}+L_{n-2}$ $(n \geq 2), L_{0}=2, L_{1}=1$, with $F_{-n}=(-1)^{n-1} F_{n}$ and $L_{-n}=(-1)^{n} L_{n}$. Exhaustive discussion of the properties of Fibonacci and Lucas numbers can be found in $[11,16]$.

The Jacobsthal numbers $J_{n}$ and the Jacobsthal-Lucas numbers $j_{n}$ are defined, for $n \in \mathbb{Z}$, through the recurrence relations $J_{n}=J_{n-1}+2 J_{n-2}(n \geq 2), J_{0}=0, J_{1}=1$ and $j_{n}=j_{n-1}+2 j_{n-2}(n \geq 2), j_{0}=2, j_{1}=1$, with $J_{-n}=(-1)^{n-1} 2^{-n} J_{n}$ and $j_{-n}=(-1)^{n} 2^{-n} j_{n}$. The entries A001045 and A014551 from [15] conclude good reference materials on the Jacobsthal and Jacobsthal-Lucas numbers, respectively.

The Pell numbers $P_{n}$ and Pell-Lucas numbers $Q_{n}$ are defined, for $n \in \mathbb{Z}$, through the recurrence relations $P_{n}=2 P_{n-1}+P_{n-2}(n \geq 2), P_{0}=0, P_{1}=1$ and $Q_{n}=2 Q_{n-1}+Q_{n-2}$ $(n \geq 2), Q_{0}=2, Q_{1}=2$, with $P_{-n}=(-1)^{n-1} P_{n}$ and $Q_{-n}=(-1)^{n} Q_{n}$. [12] and [15] (entries A000129 and A002203) are useful source materials on Pell and Pell-Lucas numbers.

For reference, the first few values of the six sequences are given in Table 1 below. 


\begin{tabular}{|c|c|c|c|c|c|c|c|c|c|c|c|c|c|c|}
\hline \hline $\boldsymbol{n}$ & -5 & -4 & -3 & -2 & -1 & 0 & 1 & 2 & 3 & 4 & 5 & 6 & 7 & 8 \\
\hline \hline $\boldsymbol{F}_{\boldsymbol{n}}$ & 5 & -3 & 2 & -1 & 1 & 0 & 1 & 1 & 2 & 3 & 5 & 8 & 13 & 21 \\
\hline $\boldsymbol{L}_{\boldsymbol{n}}$ & -11 & 7 & -4 & 3 & -1 & 2 & 1 & 3 & 4 & 7 & 11 & 18 & 29 & 47 \\
\hline $\boldsymbol{P}_{\boldsymbol{n}}$ & 29 & -12 & 5 & -2 & 1 & 0 & 1 & 2 & 5 & 12 & 29 & 70 & 169 & 408 \\
\hline $\boldsymbol{Q}_{\boldsymbol{n}}$ & -82 & 34 & -14 & 6 & -2 & 2 & 2 & 6 & 14 & 34 & 82 & 198 & 478 & 1154 \\
\hline $\boldsymbol{J}_{\boldsymbol{n}}$ & $11 / 32$ & $-5 / 16$ & $3 / 8$ & $-1 / 4$ & $1 / 2$ & 0 & 1 & 1 & 3 & 5 & 11 & 21 & 43 & 85 \\
\hline $\boldsymbol{j}_{\boldsymbol{n}}$ & $-31 / 32$ & $17 / 16$ & $-7 / 8$ & $5 / 4$ & $-1 / 2$ & 2 & 1 & 5 & 7 & 17 & 31 & 65 & 127 & 257 \\
\hline
\end{tabular}

Table 1. Terms of $F_{n}, L_{n}, P_{n}, Q_{n}, J_{n}$ and $j_{n}$

\subsection{Sum of the products of Fibonacci numbers}

\section{with subscripts in arithmetic progression}

Let $\left(X_{n}^{(1)}\right) \equiv\left(F_{n}\right)$ and $\left(X_{n}^{(2)}\right) \equiv\left(F_{n}\right)$. Then $\left(u_{n}^{(1)}\right)=\left(F_{n}\right),\left(v_{n}^{(1)}\right)=\left(L_{n}\right),\left(u_{n}^{(2)}\right)=\left(F_{n}\right)$ and $\left(v_{n}^{(2)}\right)=\left(L_{n}\right)$. Thus $p_{1}=p_{2}=1, q_{1}=q_{2}=-1$. We therefore have:

$$
\begin{gathered}
E=(-1)^{r} F_{r k+s} F_{m k+2 m+t} z^{k+2}-F_{r k+r+s} F_{m k+m+t} z^{k+1}-(-1)^{r} F_{s-r} F_{m+t} z+F_{s} F_{t}, \\
F=(-1)^{r} F_{r k+s} F_{m k+2 m+t-1} z^{k+2}-F_{r k+r+s} F_{m k+m+t-1} z^{k+1} \\
-(-1)^{r} F_{s-r} F_{m+t-1} z+F_{s} F_{t-1} \\
G=(-1)^{r} F_{2 m} z^{2}-L_{r} F_{m} z
\end{gathered}
$$

and

$$
H=(-1)^{r} F_{2 m-1} z^{2}-L_{r} F_{m-1} z+1 .
$$

Theorem 3.1. Let $r, s, m, t$ and $k$ be integers. Then

$$
\sum_{j=0}^{k} F_{r j+s} F_{m j+t} z^{j}=\frac{E H-F G}{H^{2}-G^{2}+G H},
$$

where $E, F, G$ and $H$ are as given in (11)-(14).

In particular, we have

$$
\begin{aligned}
\sum_{j=0}^{k} F_{j}^{2} z^{j} & =\frac{\left(F_{k} F_{k+2} z^{k+2}+F_{k+1}^{2} z^{k+1}-z\right)\left(1-z^{2}\right)+F_{k} F_{k+1} z^{k}\left(z+z^{2}\right)^{2}}{\left(z+z^{2}\right)^{2}-\left(1-z^{2}\right)^{2}+\left(z+z^{2}\right)\left(1-z^{2}\right)} \\
& =\frac{\left(F_{k} F_{k+2} z^{k+2}+F_{k+1}^{2} z^{k+1}-z\right)\left(z^{2}-1\right)-F_{k} F_{k+1} z^{k}\left(z+z^{2}\right)^{2}}{\left(1-3 z+z^{2}\right)(z+1)^{2}}
\end{aligned}
$$

which at $z=1$ gives the classical result

$$
\sum_{j=0}^{k} F_{j}^{2}=F_{k} F_{k+1},
$$

and from which we also get the generating function of the squares of Fibonacci numbers

$$
\sum_{j=0}^{\infty} F_{j}^{2} z^{j}=\frac{z-z^{2}}{1-2 z-2 z^{2}+z^{3}} .
$$


Evaluation of (15) at $z=-1$, with the aid of L'Hospital rule gives

$$
\sum_{j=0}^{k}(-1)^{j} F_{j}^{2}=-\frac{2}{5}(k+1)+(-1)^{k} \frac{F_{k+1} L_{k}}{5} .
$$

\subsection{Sum of the products of Fibonacci and Lucas numbers with subscripts in arithmetic progression}

Let $\left(X_{n}^{(1)}\right) \equiv\left(F_{n}\right)$ and $\left(X_{n}^{(2)}\right) \equiv\left(L_{n}\right)$. Then $\left(u_{n}^{(1)}\right)=\left(F_{n}\right),\left(v_{n}^{(1)}\right)=\left(L_{n}\right),\left(u_{n}^{(2)}\right)=\left(F_{n}\right)$ and $\left(v_{n}^{(2)}\right)=\left(L_{n}\right)$. Thus $p_{1}=p_{2}=1, q_{1}=q_{2}=-1$. We have

$$
\begin{gathered}
E=(-1)^{r} F_{r k+s} F_{m k+2 m+t} z^{k+2}-F_{r k+r+s} F_{m k+m+t} z^{k+1}-(-1)^{r} F_{s-r} F_{m+t} z+F_{s} F_{t}, \\
F=(-1)^{r} F_{r k+s} F_{m k+2 m+t-1} z^{k+2}-F_{r k+r+s} F_{m k+m+t-1} z^{k+1} \\
-(-1)^{r} F_{s-r} F_{m+t-1} z+F_{s} F_{t-1} \\
G=(-1)^{r} F_{2 m} z^{2}-L_{r} F_{m} z
\end{gathered}
$$

and

$$
H=(-1)^{r} F_{2 m-1} z^{2}-L_{r} F_{m-1} z+1 .
$$

Theorem 3.2. Let $r, s, m$, $t$ and $k$ be integers. Then

$$
\sum_{j=0}^{k} F_{r j+s} L_{m j+t} z^{j}=\frac{2(F H-E G)+E H+F G}{H^{2}-G^{2}+G H},
$$

where $E, F, G$ and $H$ are as given in (16)-(19).

In particular, we have

$$
\sum_{j=0}^{k} F_{j} L_{j} z^{j}=\frac{A+B}{\left(1-z^{2}\right)^{2}-\left(z+z^{2}\right)^{2}-\left(z+z^{2}\right)\left(1-z^{2}\right)},
$$

where

$$
\begin{aligned}
& A=-2\left(F_{k} F_{k+1} z^{k+1}(1+z)\left(1-z^{2}\right)+\left(F_{k} F_{k+2} z^{k+2}+F_{k+1}^{2} z^{k+1}-z\right)\left(z+z^{2}\right)\right), \\
& B=\left(-F_{k} F_{k+2} z^{k+2}-F_{k+1}^{2} z^{k+1}+z\right)\left(1-z^{2}\right)+F_{k} F_{k+1} z^{k+2}(1+z)^{2} .
\end{aligned}
$$

Two special evaluations are

$$
\sum_{j=0}^{k} F_{j} L_{j}=F_{2 k+1}-1
$$

which is also a classical result and

$$
\sum_{j=0}^{k} \frac{F_{j} L_{j}}{2^{j}}=\frac{2 F_{k+1}^{2}+F_{k} F_{k+3}}{2^{k}}-2 .
$$

Applying L'Hospital rule twice to the right hand side of (20) at $z=-1$ and making use of Cassini's identity, we find

$$
\sum_{j=0}^{k}(-1)^{j} F_{j} L_{j}=(-1)^{k} F_{k} F_{k+1}
$$




\subsection{Sum of the products of Fibonacci and Pell numbers with subscripts in arithmetic progression}

Let $\left(X_{n}^{(1)}\right) \equiv\left(F_{n}\right)$ and $\left(X_{n}^{(2)}\right) \equiv\left(P_{n}\right)$. Then $\left(u_{n}^{(1)}\right)=\left(F_{n}\right),\left(v_{n}^{(1)}\right)=\left(L_{n}\right),\left(u_{n}^{(2)}\right)=\left(P_{n}\right)$ and $\left(v_{n}^{(2)}\right)=\left(Q_{n}\right)$. Thus, $p_{1}=1, q_{1}=-1, p_{2}=2$ and $q_{2}=-1$. We therefore have

$$
\begin{gathered}
E=(-1)^{r} F_{r k+s} P_{m k+2 m+t} z^{k+2}-F_{r k+r+s} P_{m k+m+t} z^{k+1}-(-1)^{r} F_{s-r} P_{m+t} z+F_{s} P_{t}, \\
F=(-1)^{r} F_{r k+s} P_{m k+2 m+t-1} z^{k+2}-F_{r k+r+s} P_{m k+m+t-1} z^{k+1} \\
-(-1)^{r} F_{s-r} P_{m+t-1} z+F_{s} P_{t-1}, \\
G=(-1)^{r} P_{2 m} z^{2}-L_{r} P_{m} z
\end{gathered}
$$

and

$$
H=(-1)^{r} P_{2 m-1} z^{2}-L_{r} P_{m-1} z+1 .
$$

Theorem 3.3. Let $r, s, m, t$ and $k$ be integers. Then

$$
\sum_{j=0}^{k} F_{r j+s} P_{m j+t} z^{j}=\frac{E H-F G}{H^{2}-G^{2}+2 G H},
$$

where $E, F, G$ and $H$ are as given in (21)-(24).

In particular, we have

$$
\begin{aligned}
\sum_{j=0}^{k} F_{j} P_{j} z^{j}= & \frac{F_{k} P_{k} z^{k+4}+\left(F_{k+1} P_{k-1}-F_{k} P_{k+1}\right) z^{k+3}}{z^{4}-2 z^{3}-7 z^{2}-2 z+1} \\
& -\frac{\left(F_{k} P_{k+2}+F_{k+1} P_{k}\right) z^{k+2}+F_{k+1} P_{k+1} z^{k+1}+z^{3}-z}{z^{4}-2 z^{3}-7 z^{2}-2 z+1}
\end{aligned}
$$

of which we can mention the special values

$$
\sum_{j=0}^{k} F_{j} P_{j}=\frac{F_{k} P_{k+1}+P_{k} F_{k+1}}{3}
$$

and

$$
\sum_{j=0}^{k}(-1)^{j} F_{j} P_{j}=(-1)^{k}\left(P_{k+1} F_{k}-F_{k+1} P_{k}\right) .
$$

Note that from (25), it follows that the generating function of the product of Fibonacci numbers and Pell numbers is

$$
\sum_{j=0}^{\infty} F_{j} P_{j} z^{j}=\frac{z-z^{3}}{1-2 z-7 z^{2}-2 z^{3}+z^{4}} .
$$

\subsection{Sum of the products of Fibonacci and Jacobsthal numbers with subscripts in arithmetic progression}

Let $\left(X_{n}^{(1)}\right) \equiv\left(F_{n}\right)$ and $\left(X_{n}^{(2)}\right) \equiv\left(J_{n}\right)$. Then $\left(u_{n}^{(1)}\right)=\left(F_{n}\right),\left(v_{n}^{(1)}\right)=\left(L_{n}\right),\left(u_{n}^{(2)}\right)=\left(J_{n}\right)$ and $\left(v_{n}^{(2)}\right)=\left(j_{n}\right)$. Thus $p_{1}=1, q_{1}=-1, p_{2}=1$ and $q_{2}=-2$. We therefore have 


$$
\begin{gathered}
E=(-1)^{r} F_{r k+s} J_{m k+2 m+t} z^{k+2}-F_{r k+r+s} J_{m k+m+t} z^{k+1}-(-1)^{r} F_{s-r} J_{m+t} z+F_{s} J_{t}, \\
F=(-1)^{r} 2 F_{r k+s} J_{m k+2 m+t-1} z^{k+2}-2 F_{r k+r+s} J_{m k+m+t-1} z^{k+1} \\
-(-1)^{r} 2 F_{s-r} J_{m+t-1} z+2 F_{s} J_{t-1} \\
G=(-1)^{r} J_{2 m} z^{2}-L_{r} J_{m} z
\end{gathered}
$$

and

$$
H=(-1)^{r} 2 J_{2 m-1} z^{2}-2 L_{r} J_{m-1} z+1
$$

Theorem 3.4. Let $r, s, m, n$ and $k$ be integers. Then

$$
\sum_{j=0}^{k} F_{r j+s} J_{m j+t} z^{j}=\frac{E H-F G}{H^{2}-2 G^{2}+G H},
$$

where $E, F, G$ and $H$ are as given in (26)-(29).

In particular, we have

$$
\begin{aligned}
\sum_{j=0}^{k} F_{j} J_{j} z^{j}= & \frac{4 F_{k} J_{k} z^{k+4}+2\left(2 F_{k+1} J_{k-1}-F_{k} J_{k+1}\right) z^{k+3}}{4 z^{4}-2 z^{3}-7 z^{2}-z+1} \\
& -\frac{\left(F_{k} J_{k+2}+2 F_{k+1} J_{k}\right) z^{k+2}+F_{k+1} J_{k+1} z^{k+1}+2 z^{3}-z}{4 z^{4}-2 z^{3}-7 z^{2}-z+1}
\end{aligned}
$$

giving the special values

$$
\sum_{j=0}^{k} F_{j} J_{j}=\frac{F_{k}\left(J_{k+3}-4 J_{k}\right)+F_{k+1}\left(J_{k+2}-4 J_{k-1}\right)}{5}
$$

and

$$
\sum_{j=0}^{k}(-1)^{j} F_{j} J_{j}=(-1)^{k}\left(F_{k} J_{k+2}-F_{k+1} J_{k+1}\right)+1 .
$$

From (30) we obtain the generating function of the product of Fibonacci and Jacobsthal numbers

$$
\sum_{j=0}^{\infty} F_{j} J_{j} z^{j}=\frac{z-2 z^{3}}{1-z-7 z^{2}-2 z^{3}+4 z^{4}} .
$$

\subsection{Sum of the products of Pell and Jacobsthal numbers with subscripts in arithmetic progression}

Let $\left(X_{n}^{(1)}\right) \equiv\left(P_{n}\right)$ and $\left(X_{n}^{(2)}\right) \equiv\left(J_{n}\right)$. Then $\left(u_{n}^{(1)}\right)=\left(P_{n}\right),\left(v_{n}^{(1)}\right)=\left(Q_{n}\right),\left(u_{n}^{(2)}\right)=\left(J_{n}\right)$ and $\left(v_{n}^{(2)}\right)=\left(j_{n}\right)$. Thus $p_{1}=2, q_{1}=-1, p_{2}=1$ and $q_{2}=-2$. We therefore have

$$
E=(-1)^{r} P_{r k+s} J_{m k+2 m+t} z^{k+2}-P_{r k+r+s} J_{m k+m+t} z^{k+1}-(-1)^{r} P_{s-r} J_{m+t} z+P_{s} J_{t},
$$




$$
\begin{gathered}
F=(-1)^{r} 2 P_{r k+s} J_{m k+2 m+t-1} z^{k+2}-2 P_{r k+r+s} J_{m k+m+t-1} z^{k+1} \\
-(-1)^{r} 2 P_{s-r} J_{m+t-1} z+2 P_{s} J_{t-1} \\
G=(-1)^{r} J_{2 m} z^{2}-Q_{r} J_{m} z
\end{gathered}
$$

and

$$
H=(-1)^{r} 2 J_{2 m-1} z^{2}-2 Q_{r} J_{m-1} z+1 .
$$

Theorem 3.5. Let $r, s, m, t$ and $k$ be integers. Then

$$
\sum_{j=0}^{k} P_{r j+s} J_{m j+t} z^{j}=\frac{E H-F G}{H^{2}-2 G^{2}+G H}
$$

where E, F, G and $H$ are given in (31)-(34).

In particular, we have

$$
\begin{aligned}
\sum_{j=0}^{k} P_{j} J_{j} z^{j}= & \frac{\left(-P_{k} J_{k+2} z^{k+2}-P_{k+1} J_{k+1} z^{k+1}+z\right)\left(1-2 z^{2}\right)}{\left(4 z^{2}+4 z-1\right)\left(z^{2}-2 z-1\right)} \\
& -\frac{2\left(P_{k} J_{k+1} z^{k+2}+P_{k+1} J_{k} z^{k+1}\right)\left(2 z+z^{2}\right)}{\left(4 z^{2}+4 z-1\right)\left(z^{2}-2 z-1\right)}
\end{aligned}
$$

from which we get the special values

$$
\sum_{j=0}^{k} P_{j} J_{j}=\frac{3}{7}\left(P_{k} J_{k+1}+J_{k} P_{k+1}\right)-\frac{1}{14}\left(P_{k} J_{k+2}+J_{k+1} P_{k+1}\right)+\frac{1}{14},
$$

and

$$
\sum_{j=0}^{k}(-1)^{j} P_{j} J_{j}=\frac{(-1)^{k}}{2}\left(P_{k+1} J_{k+2}-P_{k} J_{k+3}\right)-\frac{1}{2} .
$$

From (35), we obtain the generating function of the product of Pell and Jacobsthal numbers as follows

$$
\sum_{j=0}^{\infty} P_{j} J_{j} z^{j}=\frac{z-2 z^{3}}{\left(4 z^{2}+4 z-1\right)\left(z^{2}-2 z-1\right)}
$$

\section{Conclusion}

In this paper, we have derived an expression for the partial sum of the products of two arbitrary Horadam sequences with subscripts in arithmetic progression. Illustrative examples were drawn from six well-known Horadam sequences. Some more ideas for future work were stated implicitly in the text.

\section{Acknowledgements}

The authors would like to thank the referees for their valuable comments. 


\section{References}

[1] Adegoke, K. (2018). Partial sums and generating functions for power of second order sequences with indices in arithmetic progression. Preprint. Available online at: https: //arxiv.org/abs/1904.09916.

[2] Belbachir, H., \& Bencherif, F. (2013). Sums of products of generalized Fibonacci and Lucas numbers. Ars Combinatoria, 110, 33-43.

[3] Čerin, Z. (2015). Formulas for quadratic sums that involve generalized Fibonacci and Lucas numbers. Rad Hrvatske Akademije Znanosti i Umjetnosti, Matematičke Znanosti, 19(523), $1-12$.

[4] Čerin, Z. (2009). On sum of products of Horadam numbers. Kyungpook Mathematical Journal, 49, 483-492.

[5] Čerin, Z. (2009) Sum of products of generalized Fibonacci and Lucas numbers. Demonstratio Mathematica, 42, 247-258.

[6] Horadam, A. F. (1965). Basic properties of a certain generalized sequence of numbers. The Fibonacci Quarterly, 3(3), 161-176.

[7] Khan, M. A., \& Kwong, H. (2014). On sums of products of Fibonacci-type recurrences. The Fibonacci Quarterly, 52(2), 20-26.

[8] Kiliç, E., Ömür, N., \& Ulutaş, Y. T. (2011). Some finite sums involving generalized Fibonacci and Lucas numbers. Discrete Dynamics in Nature and Society, 2011(1), Article ID 284261.

[9] Kiliç, E., \& Prodinger, H. (2015). Sums of products of generalized Fibonacci and Lucas numbers. Acta Mathematica Hungarica, 145(1), 17-25.

[10] Kiliç, E., \& Stănică, P. (2013). General approach in computing sums of products of binary sequences. Hacettepe Journal of Mathematics and Statistics, 42(1), 1-7.

[11] Koshy, T. (2017). Fibonacci and Lucas Numbers with Applications, Wiley, New York.

[12] Koshy, T. (2014). Pell and Pell-Lucas Numbers with Applications, Springer, New York.

[13] Larcombe, P. J. (2017). Horadam sequences: A survey update and extension. Bulletin of the Institute of Combinatorics and its Applications, 80, 99-118.

[14] Ribenboim, P. (2000). My Numbers, My Friends, Springer, New York.

[15] Sloane, N. J. A. (ed.). The On-Line Encyclopedia of Integer Sequences. Available online at: https://oeis.org.

[16] Vajda, S. (2008). Fibonacci \& Lucas Numbers, and the Golden Section: Theory and Applications, Dover, New York. 